PROCEEDINGS OF THE

AMERICAN MATHEMATICAL SOCIETY

Volume 130, Number 3, Pages 675-681

S 0002-9939(01)06146-9

Article electronically published on June 19, 2001

\title{
EQUALITY OF TWO SPECTRA ARISING IN HARMONIC ANALYSIS AND SEMIGROUP THEORY
}

\author{
RALPH CHILL AND EVA FAŠANGOVÁ \\ (Communicated by David R. Larson)
}

\begin{abstract}
We show that a new notion of a spectrum of a function $u \in$ $L^{\infty}\left(\mathbb{R}_{+}, X\right)$ ( $X$ is a Banach space), defined by B. Basit and the first author, coincides with the Arveson spectrum of some shift group, provided $u$ is uniformly continuous. We apply this result to prove a new version of a tauberian theorem.
\end{abstract}

In the last decade there has been an extensive study of the problem to determine the asymptotic behaviour of a bounded and uniformly continuous function $u: \mathbb{R}_{+} \rightarrow$ $X$ ( $X$ a Banach space) via tauberian theorems; cf. [3, 7] for accounts of this theory and its applications to evolution equations. There are mainly two approaches to this problem: the operator theoretical approach by $C_{0}$-semigroups and -groups and the approach by harmonic analysis (Fourier transforms). In both approaches, the tauberian conditions are of spectral type. It is the purpose of this note to show that two spectra from both approaches actually coincide, and that in this sense the two approaches are equivalent. We also restate the main tauberian theorem from 2, 5, 7] in a form which is similar to Loomis' theorem about almost periodic functions, as it is stated in Katznelson's book [9] Theorem 5.20 and Ex. 7, p.169] (see also Loomis' original article [10]).

We will denote by $S$ the semigroup of translations on $B U C\left(\mathbb{R}_{+}, X\right)$ equipped with the topology of uniform convergence (i.e. $S(t) u:=u(t+\cdot)$ ), and the infinitesimal generator of $S$ will be denoted by $D$.

Definition $1([2])$. A set $\mathcal{E} \subset B U C\left(\mathbb{R}_{+}, X\right)$ is called $S$-biinvariant if the following conditions are satisfied:

(i) $\mathcal{E}$ is a closed subspace of $B U C\left(\mathbb{R}_{+}, X\right)$,

(ii) if $t \geq 0$ and $u \in \mathcal{E}$, then $S(t) u \in \mathcal{E}$,

(iii) if $t \geq 0$ and $u \in B U C\left(\mathbb{R}_{+}, X\right)$ is such that $S(t) u \in \mathcal{E}$, then $u \in \mathcal{E}$.

It follows from condition (iii) that every $S$-biinvariant subspace $\mathcal{E}$ contains the compactly supported functions and therefore, by closedness, the space $C_{0}\left(\mathbb{R}_{+}, X\right)$.

Received by the editors February 7, 2000 and, in revised form, August 2, 2000.

2000 Mathematics Subject Classification. Primary 47D03; Secondary 47A10, $40 \mathrm{E} 05$.

Key words and phrases. Spectrum of a function, Arveson spectrum, $C_{0}$-group, Fourier transform, tauberian theorem.

Part of this work was done while the first author visited the Charles University of Prague. He is grateful for the warm hospitality and the financial support. The second author is supported by grant No. 201/98/1450 of the Grant Agency of the Czech Republic, grant No. 166/1999 of the Grant Agency of Charles University, and grant No. CEZ J 13/98113200007.

(C)2001 American Mathematical Society 
Examples of $S$-biinvariant subspaces $\mathcal{E} \subset B U C\left(\mathbb{R}_{+}, X\right)$ are $C_{0}\left(\mathbb{R}_{+}, X\right)$, the space $A A P\left(\mathbb{R}_{+}, X\right)$ of all asymptotically almost periodic functions, and the space of all Eberlein weakly almost periodic functions $W\left(\mathbb{R}_{+}, X\right)$. See [2, 3] 5, 7] for these concepts and more examples.

For an $S$-biinvariant subspace $\mathcal{E} \subset B U C\left(\mathbb{R}_{+}, X\right)$ we will denote the quotient space by $B U C\left(\mathbb{R}_{+}, X\right)_{/ \mathcal{E}}$, the quotient map by $\Pi: B U C\left(\mathbb{R}_{+}, X\right) \rightarrow B U C\left(\mathbb{R}_{+}, X\right)_{/ \mathcal{E}}$, and the quotient semigroup defined by $S_{\mathcal{E}}(t) \Pi u:=\Pi S(t) u$ by $S_{\mathcal{E}}$. The quotient semigroup $S_{/ \mathcal{E}}$ is well defined since $S$ leaves $\mathcal{E}$ invariant.

Lemma 2. The operator $S_{/ \mathcal{E}}(t)$ is an isometric isomorphism for all $t \geq 0$.

Proof. Let $t \geq 0$. Then the operator $S_{\mathcal{L}}(t)$ is surjective since $\Pi$ and $S(t)$ are surjective. In order to see that it is isometric, we let $f \in B U C(\mathbb{R} ; X)$. By definition

$$
\left\|S_{/ \mathcal{E}}(t) \Pi f\right\|=\|\Pi S(t) f\|=\inf _{g \in \mathcal{E}}\|S(t) f-g\|_{\infty} .
$$

Given $g \in \mathcal{E}$ we put

$$
\tilde{g}(s):= \begin{cases}g(s-t), & s \geq t, \\ f(s)-f(t)+g(0), & 0 \leq s \leq t .\end{cases}
$$

Then $S(t) \tilde{g}=g \in \mathcal{E}$, and the $S$-biinvariance of $\mathcal{E}$ implies $\tilde{g} \in \mathcal{E}$.

From this we obtain first that $S(t)$ is surjective from $\mathcal{E}$ onto $\mathcal{E}$, so that

$$
\inf _{g \in \mathcal{E}}\|S(t) f-g\|_{\infty}=\inf _{g \in \mathcal{E}}\|S(t) f-S(t) g\|_{\infty} \leq \inf _{g \in \mathcal{E}}\|f-g\|_{\infty}=\|\Pi f\| .
$$

Second, we obtain

$$
\begin{aligned}
\|S(t) f-g\|_{\infty} & =\sup _{s \geq 0}\|f(t+s)-g(s)\| \\
& =\max \left(\sup _{s \geq 0}\|f(t+s)-g(s)\|,\|f(t)-g(0)\|\right) \\
& =\max \left(\sup _{s \geq 0}\|f(t+s)-\tilde{g}(t+s)\|, \sup _{0 \leq s \leq t}\|f(s)-\tilde{g}(s)\|\right) \\
& =\sup _{s \geq 0}\|f(s)-\tilde{g}(s)\|=\|f-\tilde{g}\|_{\infty},
\end{aligned}
$$

which implies

$$
\inf _{g \in \mathcal{E}}\|S(t) f-g\|_{\infty} \geq \inf _{g \in \mathcal{E}}\|f-g\|_{\infty}=\|\Pi f\| .
$$

By Lemma 2 the semigroup $S_{/ \varepsilon}$ extends to a $C_{0}$-group of isometries. We denote its generator by $D_{/ \mathcal{E}}$.

Definition 3 ([5, 7]). Let $\mathcal{E}$ be $S$-biinvariant and let $u \in L^{\infty}\left(\mathbb{R}_{+}, X\right)$. We define the spectrum of $u$ with respect to $\mathcal{E}$ by

$$
\begin{aligned}
\operatorname{Sp}_{\mathcal{E}}^{\mathbb{R}_{+}}(u):=\{\eta \in \mathbb{R}: & \text { for every } \varepsilon>0 \text { there exists } f \in L^{1}(\mathbb{R}) \text { such that } \\
& \left.\operatorname{supp} \tilde{f} \subset(\eta-\varepsilon, \eta+\varepsilon) \text { and }\left.f \star u\right|_{\mathbb{R}_{+}} \notin \mathcal{E}\right\} ;
\end{aligned}
$$

here $f \star u(t):=\int_{0}^{\infty} f(t-s) u(s) d s$ and $\tilde{f}(\varrho):=\int_{-\infty}^{+\infty} e^{-i \varrho s} f(s) d s$. 
Definition $4\left([1,2,[3])\right.$. Let $T$ be a bounded $C_{0}$-group on a Banach space $Y$. The local Arveson spectrum of some $y \in Y$ with respect to $T$ is defined by

$\operatorname{Sp}^{A}(T(\cdot) y):=\left\{\eta \in \mathbb{R} \quad: \quad\right.$ for every $\varepsilon>0$ there exists $f \in L^{1}(\mathbb{R})$ such that

$$
\left.\operatorname{supp} \tilde{f} \subset(-\eta-\varepsilon,-\eta+\varepsilon) \text { and } \int_{-\infty}^{+\infty} f(s) T(s) y d s \neq 0\right\} \text {, }
$$

whereas the Arveson spectrum of $T$ is defined by

$\operatorname{Sp}^{A}(T):=\left\{\eta \in \mathbb{R} \quad: \quad\right.$ for every $\varepsilon>0$ there exist $y \in Y$ and $f \in L^{1}(\mathbb{R})$ such that

$$
\left.\operatorname{supp} \tilde{f} \subset(-\eta-\varepsilon,-\eta+\varepsilon) \text { and } \int_{-\infty}^{+\infty} f(s) T(s) y d s \neq 0\right\} \text {. }
$$

It follows immediately from Definitions 3 and 4 that the spectra defined there are closed subsets of $\mathbb{R}$.

By definition of the Arveson spectrum of a group $T$ on a Banach space $Y$ we have the following equality:

$$
\mathrm{Sp}^{A}(T)=\overline{\bigcup_{y \in Y} \operatorname{Sp}^{A}(T(\cdot) y)}
$$

(here, the proof of the inclusion $\operatorname{Sp}^{A}(T) \supset \overline{\bigcup_{y \in Y} \operatorname{Sp}^{A}(T(\cdot) y)}$ is elementary and for the opposite inclusion one has to use a partition of unity argument).

Moreover, it is well known (cf. for example [8, Proposition IV.3.18]) that the Arveson spectrum of a $C_{0}$-group $T$ coincides, up to a factor $i$, with the spectrum of its generator $B$, i.e. we have

$$
i \cdot \mathrm{Sp}^{A}(T)=\sigma(B)
$$

(Note that the definition of the Arveson spectrum in [8] differs from ours, but it can be shown, using e.g. the Wiener-Lévy theorem ([11] Section I.3.1]), that the two definitions are equivalent up to a factor -1 .)

The following is the main theorem of this note.

Theorem 5. Let $\mathcal{E} \subset B U C\left(\mathbb{R}_{+}, X\right)$ be $S$-biinvariant and let $u \in B U C\left(\mathbb{R}_{+}, X\right)$. Then

$$
\operatorname{Sp}_{\mathcal{E}}^{\mathbb{R}_{+}}(u)=\operatorname{Sp}^{A}\left(\left(S_{/ \mathcal{E}}\right)_{\Pi u}\right),
$$

where $\left(S_{/ \mathcal{E}}\right)_{\Pi u}$ is the restriction of the quotient $S_{/ \mathcal{E}}$ of the shift semigroup $S$ to the closed linear span of the complete orbit of $\Pi u$, i.e. to $\overline{\operatorname{span}}\left\{S_{/ \mathcal{E}}(t) \Pi u, t \in \mathbb{R}\right\} \subset$ $B U C\left(\mathbb{R}_{+}, X\right)_{/ \mathcal{E}}$.

Proof. For $s \leq 0$ we define an operator $S(s)$ on $B U C\left(\mathbb{R}_{+}, X\right)$ by

$$
S(s) v(t):=\left\{\begin{array}{ll}
v(0), & t+s<0, \\
v(t+s), & t+s \geq 0,
\end{array} \quad t \in \mathbb{R}_{+}, v \in B U C\left(\mathbb{R}_{+}, X\right) .\right.
$$

Then $S(-s)$ is an isometric right inverse of $S(s)$ for all $s \geq 0$, and for all $s \in \mathbb{R}$ the $S$-biinvariance of $\mathcal{E}$ implies

$$
S_{/ \mathcal{E}}(s) \Pi v=\Pi S(s) v .
$$


Moreover, by definition of the quotient group and by linearity and continuity of $\Pi$ we have

$$
\begin{aligned}
& \left(B U C\left(\mathbb{R}_{+}, X\right)_{/ \mathcal{E}}\right)_{\Pi u}:=\overline{\operatorname{span}}\left\{S_{/ \mathcal{E}}(t) \Pi u: t \in \mathbb{R}\right\} \\
& =\overline{\operatorname{span}}\{\Pi S(t) u: t \in \mathbb{R}\} \\
& =\overline{\Pi \operatorname{span}\{S(t) u: t \in \mathbb{R}\}} \text {. }
\end{aligned}
$$

Hence, for any $v \in \operatorname{span}\{S(t) u: t \in \mathbb{R}\}$ and any $f \in L^{1}(\mathbb{R})$ the following integral belongs to $\left(B U C\left(\mathbb{R}_{+}, X\right)_{/ \mathcal{E}}\right)_{\Pi u}$ :

$$
\begin{aligned}
\int_{-\infty}^{+\infty} f(s) S_{/ \mathcal{E}}(s) \Pi v d s & =\int_{-\infty}^{+\infty} f(s) \Pi S(s) v d s=\Pi \int_{-\infty}^{+\infty} f(s) S(s) v d s \\
& =\Pi \int_{-\infty}^{+\infty} f(s-\cdot)(S(s) v)(0) d s \\
& =\Pi\left(\int_{-\infty}^{0} f(s-\cdot) v(0) d s+\int_{0}^{+\infty} f(s-\cdot) v(s) d s\right) \\
& =\left.\Pi(g \star v)\right|_{\mathbb{R}_{+} .}
\end{aligned}
$$

In the last step we have used the property $C_{0}\left(\mathbb{R}_{+}, X\right) \subset \mathcal{E}$ and we have put $g(s):=f(-s)$.

Now, if $\eta \in \operatorname{Sp}_{\mathcal{E}}^{\mathbb{R}_{+}}(u)$, then for every $\varepsilon>0$ there exists $g \in L^{1}(\mathbb{R})$ such that $\operatorname{supp} \tilde{g} \subset(\eta-\varepsilon, \eta+\varepsilon)$ and $\left.(g \star u)\right|_{\mathbb{R}_{+}} \notin \mathcal{E}$. By the above equation this implies, for $f(s):=g(-s)$,

$$
\int_{-\infty}^{+\infty} f(s) S_{/ \mathcal{E}}(s) \Pi u d s \neq 0
$$

Noting that $\operatorname{supp} \tilde{f}=-\operatorname{supp} \tilde{g}$ we therefore obtain $\eta \in \operatorname{Sp}^{A}\left(\left(S_{/ \mathcal{E}}\right)_{\Pi u}\right)$.

If, conversely, $\eta \in \operatorname{Sp}^{A}\left(\left(S_{/ \mathcal{E}}\right)_{\Pi u}\right)$, then for every $\varepsilon>0$ there exist an $f \in L^{1}(\mathbb{R})$ and, by density, an element $v \in \operatorname{span}\{S(t) u: t \in \mathbb{R}\}$ such that

$$
\operatorname{supp} \tilde{f} \subset(-\eta-\varepsilon,-\eta+\varepsilon)
$$

and

$$
\int_{-\infty}^{+\infty} f(s) S_{/ \mathcal{E}}(s) \Pi v d s \neq 0 .
$$

By the above equation again this implies $\left.(g \star v)\right|_{\mathbb{R}_{+}} \notin \mathcal{E}$, and it remains to show that this is equivalent to $\left.(g \star u)\right|_{\mathbb{R}_{+}} \notin \mathcal{E}$. To prove this last step, we proceed by contradiction. Suppose that $\left.(g \star u)\right|_{\mathbb{R}_{+}} \in \mathcal{E}$. Let first $v:=S(s) u(s \in \mathbb{R})$. Then the function

$$
\begin{aligned}
(g \star v)(t) & =\int_{0}^{+\infty} g(t-z)(S(s) u)(z) d z \\
& =\int_{s}^{+\infty} g(t+s-z)(S(z) u)(0) d z \\
& =(g(\cdot+s) \star u)(t)+\int_{s}^{0} g(t+s-z)(S(z) u)(0) d z \\
& =(g \star u)(t+s)+\int_{s}^{0} g(t+s-z)(S(z) u)(0) d z
\end{aligned}
$$


restricted to $\mathbb{R}_{+}$is in $\mathcal{E}$ since $\mathcal{E}$ is $S$-biinvariant and $\int_{s}^{0} g(\cdot+s-z)(S(z) u)(0) d z \in$ $C_{0}\left(\mathbb{R}_{+}, X\right) \subset \mathcal{E}$. By linearity we obtain the desired conclusion for any $v$ in the $\operatorname{span}\{S(t) u: t \in \mathbb{R}\}$. Hence, $\eta \in \operatorname{Sp}_{\mathcal{E}}^{\mathbb{R}_{+}}(u)$.

The following known result may be obtained directly from Theorem 5 and equation (11).

Corollary 6. Let $\mathcal{E}$ and $u$ be as in Theorem 5 . Then

$$
i \cdot \operatorname{Sp}_{\mathcal{E}}^{\mathbb{R}_{+}}(u)=\sigma\left(\left(D_{/ \mathcal{E}}\right)_{\Pi u}\right),
$$

where $\left(D_{/ \mathcal{E}}\right)_{\Pi u}$ is the part of the generator of the quotient group $S_{/ \mathcal{E}}$ in the space $\overline{\operatorname{span}}\left\{S_{/ \mathcal{E}}(t) \Pi u: t \in \mathbb{R}\right\}$.

Remark 7. There is an analogous statement of Corollary 6] in [6] page 321] (see also [1, proof of Theorem 3.4]) for functions defined on the line. The relation between [6] and Corollary [6] can be described as follows.

For a given $S$-biinvariant space $\mathcal{E} \subset B U C\left(\mathbb{R}_{+}, X\right)$ we set $\mathcal{E}_{1}:=\{u \in B U C(\mathbb{R}, X)$; $\left.\left.u\right|_{\mathbb{R}_{+}} \in \mathcal{E}\right\}$. Then $\mathcal{E}_{1} \subset B U C(\mathbb{R}, X)$ is closed and translation-invariant in the sense that $\mathcal{E}_{1}=\left\{f(t+\cdot): f \in \mathcal{E}_{1}\right\}$ for every $t \in \mathbb{R}$.

Extending functions $u \in L^{\infty}\left(\mathbb{R}_{+}, X\right)$ by 0 on $\mathbb{R}_{-}$, it is easy to check that

$$
\operatorname{Sp}_{\mathcal{E}}^{\mathbb{R}_{+}}(u)=\operatorname{Sp}_{\mathcal{E}_{1}}^{\mathbb{R}}(u),
$$

where the line spectrum with respect to $\mathcal{E}_{1}$ is defined as in [5] 6] by

$$
\begin{aligned}
\operatorname{Sp}_{\mathcal{E}_{1}}^{\mathbb{R}}(u):=\{\eta \in \mathbb{R}: & \text { for every } \varepsilon>0 \text { there exists } f \in L^{1}(\mathbb{R}) \text { such that } \\
& \left.\operatorname{supp} \tilde{f} \subset(\eta-\varepsilon, \eta+\varepsilon) \text { and } f \star u \notin \mathcal{E}_{1}\right\} .
\end{aligned}
$$

Hence, Corollary 6 follows also from [6] and [1. Note that by this approach Corollary 6] appears to be weaker than the statement in [6, page 321], since not every translation-invariant space $\mathcal{E}_{1} \subset B U C(\mathbb{R}, X)$ comes from an $S$-biinvariant space in the above way. A simple counterexample is the space $\mathcal{E}_{1}=\{0\}$.

Using our characterization of the spectrum $\operatorname{Sp}_{\mathcal{E}}^{\mathbb{R}_{+}}(u)$ we can reformulate the tauberian theorems [7, Theorem 1.19] and [2] Theorem 2.3] (where a different, larger spectrum is used; cf. equation (44). Let us point out that the countability condition from [2, 7] is dropped; we rather state that under some ergodicity condition the spectrum $\operatorname{Sp}_{\mathcal{E}}^{\mathbb{R}_{+}}(u)$ does not contain an isolated point.

Definition 8 ([1]). Let $u \in B U C\left(\mathbb{R}_{+}, X\right)$ and $\eta \in \mathbb{R}$. We say that $u$ is uniformly ergodic at $\eta$ if the following limit (called mean) exists in $B U C\left(\mathbb{R}_{+}, X\right)$ :

$$
M_{\eta}(u):=\lim _{\alpha \rightarrow 0_{+}} \alpha \int_{0}^{\infty} e^{-(\alpha+i \eta) t} S(t) u d t .
$$

We call $u$ totally uniformly ergodic if it is uniformly ergodic at any $\eta \in \mathbb{R}$.

It follows easily from the definition that if $u$ is uniformly ergodic at $\eta$, then the mean is an exponential function:

$$
M_{\eta}(u)(s)=e^{i \eta s} M_{\eta}(u)(0), \quad s \in \mathbb{R}_{+} .
$$

Moreover, on its maximal domain, the operator $M_{\eta}$ is a projection onto the eigenspace $N(i \eta-D)$. 
Theorem 9. Let $u \in B U C\left(\mathbb{R}_{+}, X\right)$ be totally ergodic. Let $\mathcal{E} \subset B U C\left(\mathbb{R}_{+}, X\right)$ be $S$-biinvariant and such that it contains $M_{\eta}(u)$ for every $\eta \in \mathbb{R}$. Then $\operatorname{Sp}_{\mathcal{E}}^{\mathbb{R}_{+}}(u)$ does not contain an isolated point.

Proof. By contradiction, suppose that $\operatorname{Sp}_{\mathcal{E}}^{\mathbb{R}_{+}}(u)$ is nonempty and contains an isolated point $\eta$. Then $i \eta$ is an isolated point of $\sigma\left(\left(D_{/ \mathcal{E}}\right)_{\Pi u}\right)$ by Corollary [6, so it is an eigenvalue by Gelfand's theorem [4], 3. Corollary 4.4.9], 8, Theorem B.17] (the assumptions are satisfied due to Lemma 2). Let $\bar{w} \in\left(B U C\left(\mathbb{R}_{+}, X\right)_{/ \mathcal{E}}\right)_{\Pi u}$ be a corresponding eigenvector. Then

$$
S_{/ \mathcal{E}}(t) \bar{w}=e^{i \eta t} \bar{w}, \quad t \in \mathbb{R}_{+} .
$$

By equation (3), we find a sequence $\left(w_{n}\right)_{n \in \mathbb{N}} \subset \operatorname{span}\{S(t) u: t \in \mathbb{R}\}$ (where $S(t)$ for $t \leq 0$ is defined as in the proof of Theorem 5uch that $\lim _{n \rightarrow \infty} \Pi w_{n}=\bar{w}$. This yields

$$
\begin{aligned}
\lim _{n \rightarrow \infty} \Pi S(t) w_{n} & =\lim _{n \rightarrow \infty} S_{/ \mathcal{E}}(t) \Pi w_{n} \\
& =S_{/ \mathcal{E}}(t) \bar{w}=e^{i \eta t} \bar{w} \\
& =e^{i \eta t} \lim _{n \rightarrow \infty} \Pi w_{n}=\lim _{n \rightarrow \infty} \Pi\left(e^{i \eta t} w_{n}\right)
\end{aligned}
$$

uniformly in $t \in \mathbb{R}_{+}$, and hence

$$
\begin{aligned}
& \left\|\alpha \int_{0}^{\infty} e^{-(\alpha+i \eta) t} \Pi\left(S(t) w_{n}-e^{i \eta t} w_{n}\right) d t\right\| \\
& \leq \alpha \int_{0}^{\infty} e^{-\alpha t} d t \sup _{t \geq 0}\left\|\Pi\left(S(t) w_{n}-e^{i \eta t} w_{n}\right)\right\|
\end{aligned}
$$

converges to 0 as $n \rightarrow \infty$, uniformly for $\alpha>0$. So

$$
\begin{aligned}
0 & =\lim _{\alpha \rightarrow 0+} \lim _{n \rightarrow \infty} \alpha \int_{0}^{+\infty} e^{-(\alpha+i \eta) t} \Pi\left(S(t) w_{n}-e^{i \eta t} w_{n}\right) d t \\
& =\lim _{n \rightarrow \infty} \lim _{\alpha \rightarrow 0+} \alpha \int_{0}^{+\infty} e^{-(\alpha+i \eta) t} \Pi\left(S(t) w_{n}-e^{i \eta t} w_{n}\right) d t \\
& =\lim _{n \rightarrow \infty}\left(\Pi \lim _{\alpha \rightarrow 0+} \alpha \int_{0}^{\infty} e^{-(\alpha+i \eta) t} S(t) w_{n} d t-\Pi w_{n}\right) \\
& =\lim _{n \rightarrow \infty} \Pi M_{\eta}\left(w_{n}\right)-\bar{w}=-\bar{w},
\end{aligned}
$$

where in the last step we have used that $M_{\eta}\left(w_{n}\right) \in \operatorname{span}\left\{M_{\eta}(S(t) u): t \in \mathbb{R}\right\}=$ $\operatorname{span}\left\{S(t)\left(M_{\eta}(u)\right): t \in \mathbb{R}\right\} \subset \mathcal{E}$. This is a contradiction to $\bar{w}$ being an eigenvector.

If for $u \in B U C\left(\mathbb{R}_{+}, X\right)$ we define the spectrum (see [2], 3, Section 4.4])

$\mathrm{Sp}^{\mathbb{R}_{+}}(u):=\{\eta \in \mathbb{R}: \widehat{u}$ has no holomorphic extension to a neighbourhood of $i \eta\}$,

where $\widehat{u}$ denotes the Laplace transform of $u$, then the following result from 2 is a corollary of Theorem 9 .

Theorem 10 (2, Theorem 2.3]). Let $u \in B U C\left(\mathbb{R}_{+}, X\right)$ be totally ergodic. Let $\mathcal{E} \subset B U C\left(\mathbb{R}_{+}, X\right)$ be $S$-biinvariant and such that it contains $M_{\eta}(u)$ for every $\eta \in$ $\mathrm{Sp}^{\mathbb{R}_{+}}(u)$. If $\mathrm{Sp}^{\mathbb{R}_{+}}(u)$ is countable, then $u \in \mathcal{E}$. 
Proof. By [7, Lemma 1.15, Lemma 1.16], we have $\operatorname{Sp}_{\mathcal{E}}^{\mathbb{R}_{+}}(u) \subset \operatorname{Sp}_{C_{0}}^{\mathbb{R}_{+}}(u) \subset \operatorname{Sp}^{\mathbb{R}_{+}}(u)$, and $M_{\eta}(u)=0$ for every $\eta \notin \mathrm{Sp}^{\mathbb{R}_{+}}(u)$, so that the assumptions of Theorem 9 are satisfied.

Hence, $\operatorname{Sp}_{\mathcal{E}}^{\mathbb{R}_{+}}(u)$ does not contain an isolated point. Since the spectrum $\operatorname{Sp}_{\mathcal{E}}^{\mathbb{R}_{+}}(u)$ is at most countable by assumption, it must be empty, since every nonempty, closed countable subset of $\mathbb{R}$ contains an isolated point. The claim $u \in \mathcal{E}$ follows from $[5$. Theorem 4.2.6] or [7, Lemma 1.15].

\section{ACKNOWLEDGEMENT}

We thank the referee for his comments on the basis of which Remark 7 was added.

\section{REFERENCES}

[1] W. Arendt and C.J.K. Batty. Almost periodic solutions of first- and second-order Cauchy problems. J. Diff. Eq. 137, 363-383, 1997. MR 98g:34099

[2] W. Arendt and C.J.K. Batty. Asymptotically almost periodic solutions of inhomogeneous Cauchy problems on the half-line. Bull. London Math. Soc. 31, 291-304, 1999. CMP 99:09

[3] W. Arendt, C.J.K. Batty, M. Hieber and F. Neubrander. Vector-valued Laplace transforms and Cauchy problems. Monographs in Mathematics, 96, Birkhäuser Verlag, Basel, 2001.

[4] W. Arveson. The harmonic analysis of automorphism groups. Operator Algebras and Applications. Proc. Symp. Pure Math. 38, Kingston/Ont. (1980), 199-269, 1982. MR 84m:46085

[5] B. Basit. Some problems concerning different types of vector valued almost periodic functions. Dissertationes Math. 338, 1995. MR 96d:43007

[6] C.J.K. Batty, W. Hutter, F. Räbiger. Almost periodicity of mild solutions of inhomogeneous periodic Cauchy problems. J. Diff. Eq. 156, 309-327, 1999. CMP 99:17

[7] R. Chill. Fourier transforms and asymptotics of evolution equations. PhD Thesis, Universität Ulm, 1998.

[8] K.-J. Engel and R. Nagel. One-Parameter Semigroups for Linear Evolution Equations. Springer Verlag, New York, Berlin, Heidelberg, 1999. MR 2000i:47075

[9] Y. Katznelson. An Introduction to Harmonic Analysis. Wiley, New York, London, 1968. MR 40:1734

[10] L. H. Loomis. The spectral characterization of a class of almost periodic functions. Ann. of Math. 72, 362-368, 1960. MR 22:11255

[11] H. Reiter. Classical Harmonic Analysis and Locally Compact Groups. Oxford Mathematical Monographs, Oxford, At the Clarendon Press, 1968. MR 46:5933

Abteilung Angewandte Analysis, Universität Ulm, 89069 Ulm, Germany

E-mail address: chill@mathematik.uni-ulm.de

Department of Mathematical Analysis, Charles University, Sokolovská 83, 18675 Praha 8, Czech Republic

E-mail address: fasanga@karlin.mff.cuni.cz 